\title{
Comparison of Odiosoft-Rhino and rhinomanometry in evaluation of nasal patency*
}

\author{
Rauf Tahamiler, Yalçin Alimoglu, Salih Canakcioglu \\ Department of Otorhinolaryngology, Istanbul University, Cerrahpasa Medical School, Istanbul, Turkey
}

SUMMARY

\begin{abstract}
Objective: To conduct a preliminary study testing the validity of the Odiosoft-Rhino (OR) in the evaluation of nasal patency in patients with nasal septal deviation and compare the results with visual analogue score (VAS) of nasal obstruction and rhinomanometry (RMM).

Methods: OR and RMM were performed on 68 patients with nasal septal deviation and 61 healthy controls. VAS, nasal resistance values and nasal sound analysis were noted for the left and right nasal cavities.

Results: There are statistically significant differences between VAS, RMM and nasal endoscopic findings between the patient and the control group. The OR results at expiratory and inspiratory 2000-4000 Hz and 4000-6000 Hz intervals for both nasal cavities show a statistically significant difference between the patient and control groups. Nasal endoscopic findings, $R M M, O R$ results of the deviated sides in the 2000-4000 Hz interval correlate well with $V A S$.

Conclusions: OR may be a practical test in assessment of nasal patency. OR at the 2000-4000 $H z$ interval shows a correlation with VAS. However, showing a correlation between a test and a variable is not the same as proving that it is a useful diagnostic test. Therefore, more studies with larger series are needed.
\end{abstract}

Key words: nasal patency, nasal septum deviation, rhinomanometry, Odiosoft-Rhino.

\section{INTRODUCTION}

Objective assessment of the nasal airway is one of the most common problems in the otolaryngology practice. There have been studies concerning respiratory sound analysis in the upper airways ${ }^{(1)}$. However, few studies about nasal acoustic analysis have been made. A recently developed method using nasal sound as a method to evaluate nasal patency by automatically analyzing the spectral parameters of nasal sound called Odisoft-Rhino (OR) may provide useful data.

In this study, we compared the OR, Rhinomanometry (RMM) and Visual analogue scale (VAS) of nasal obstruction.

\section{MATERIAL AND METHODS}

\section{Patient characteristics}

This study was performed with 68 patients who had a nasal septal deviation and nasal obstruction complaints but no symptoms of rhinitis as the patient group and 61 healthy people as a control group. The patients had symptoms of nasal obstruction on both sides of their nose.

In total, 132 patients with a nasal septal deviation were selected in a randomized fashion from the archives of examination cards and asked to volunteer in this study. Patients with a history of nasal trauma or nasal surgery and those on medication were not included in the study. Patients complaining of one-sided nasal obstruction, having severe nasal deviation having a significant difference between the nasal passages of two nostrils were also not included in the study. Nasal smear and skin prick test had been performed to all patients previously. Finally, allergic rhinitis patients were not included. According to these criteria, 68 patients were included in the patient group. The healthy control group was selected from patients admitted to our clinic due to minor complaints not related to nasal obstruction or septal deviation. Patients on medication were not included in the healthy control group. Skin prick test, nasal smear examination and VAS recording were also performed to the control group.

This study was performed with the permission of medical research ethic committee of our University and informed consent of the subjects.

\section{Nasal tests}

Nasal endoscopy was performed and any crests or spurs at the site of the septal deviation was noted (anterior, posterior, both anterior and posterior). A VAS of nasal obstruction was recorded on a diary card in both groups during 15 days. The mean value of VAS was noted for each patient. RMM was also performed on both groups.

On the VAS, patients were asked to answer the question "Could you grade the severity of nasal obstruction between 0 - 
100 ?" The diary card used contains a horizontally placed 100 $\mathrm{mm}$ line where each patient graded his or her symptom severity at the same time of the day. The symptom severity scores were noted by the patient on the card and were given to the physicians before RMM and OR.

All steps of subject selection and the tests (physical examination, endoscopy, anterior rhinomanometry, Odiosoft-Rhino) for each person were performed by different authors, individually. Each test was repeated by another physician. All physicians were instructed to perform the test the same as the others. The results were not compared until the study was finished.

RMM and OR were performed in a quiet room with a temperature of $22^{\circ} \mathrm{C}-25^{\circ} \mathrm{C}$ and a humidity ratio of $50-60 \%$. The patients were taken to the room 20 minutes before the test and let to sit on a chair until the test so they could relax. Before starting the test, all subjects were examined with nasal endoscopy and all crusts and secretions in the nasal cavities were removed taking care not to touch the nasal mucosa. The patients were told not to consume foods or drinks containing caffeine or methylxanthines and not to smoke 4 hours before the test.

OR is actually a computer program that analyzes nasal sounds. The equipment is composed of a microphone and a sound card connected to a personal computer. The microphone (diameter $18 \mathrm{~mm}$, frequency range $50 \mathrm{~Hz}-20 \mathrm{kHz}$ ) with a 2-cm-long nasal probe attached, has a low pass filter at cutoff frequency of 10 $\mathrm{kHz}$, a high pass filter at $150 \mathrm{~Hz}$ and amplifies the sound by 20 $\mathrm{dB}$. The microphone is connected to the sound card (Yamaha AC-XG WDM Audio, Japan) of the computer. Sound is received by the sound card as analogue signals, which are then converted to digital signals by a 16-bit analog-to-digital converter. Reception is made by default at $44,100 \mathrm{bits} / \mathrm{sec}$ frequency. Digital signals are then saved as wave files in the computer. The software analyses the digital data using the Fast Fourier Transform method and performs spectral analysis of the sound. The Fast Fourier Transform method has been also used in evaluation of other body sounds such as pulmonary auscultation sounds, wheezing and obstructive sleep disordered breathing ${ }^{(2-4)}$. The results of these analyses are expressed as amplitude $(\mathrm{dB})$ in the frequency intervals of $200-500 \mathrm{~Hz}$, $500-1000 \mathrm{~Hz}, 1000-2000 \mathrm{~Hz}, 2000-4000 \mathrm{~Hz}$ and 4000-6000 Hz. Artifacts like computer fan noise and the like are removed with the help of spectrogram overview FFT.

During the test, four consecutive inspirations and expirations were held and then the inspiration and expiration that had the best sound sample rate was chosen for evaluation. While testing on one nostril, the opposite nostril was gently sealed with an adhesive tape. The probe was held parallel to the tested nasal cavity and was fixed at a distance of $1 \mathrm{~cm}$ from the nostril. The recording started during non forced nasal expiration and was performed for at least 2-3 seconds. No decongestant was used during OR in our study.

In active anterior RMM (Rhinometrics, Rhinostream v2.6, ed.1.1, Interacoustics A/S, Denmark), both nasal cavities were tested without using any topical nasal decongestants. The opposite nasal cavity was gently occluded with a tape. A mask fitting the patient's face was used. While the patient was breathing through the nose, measurements were recorded. Three recordings for each nasal cavity were made, the arithmetical mean values of inspiratory and expiratory airflow at $150 \mathrm{~Pa}$ transnasal pressure were noted and resistance values were calculated.

VAS, RMM and OR results of both nasal cavities were separately noted. A comparison with the healthy control group and a correlation inquiry was made.

\section{Statistical analysis}

All data were expressed as mean value \pm standard deviation. A $\mathrm{p}$ value of less than 0.05 was considered significant. Student's t-test, Levene's test for equality of variances and Pearson correlation test were used.

Table 1. RMM and OR results of the patient and control groups.

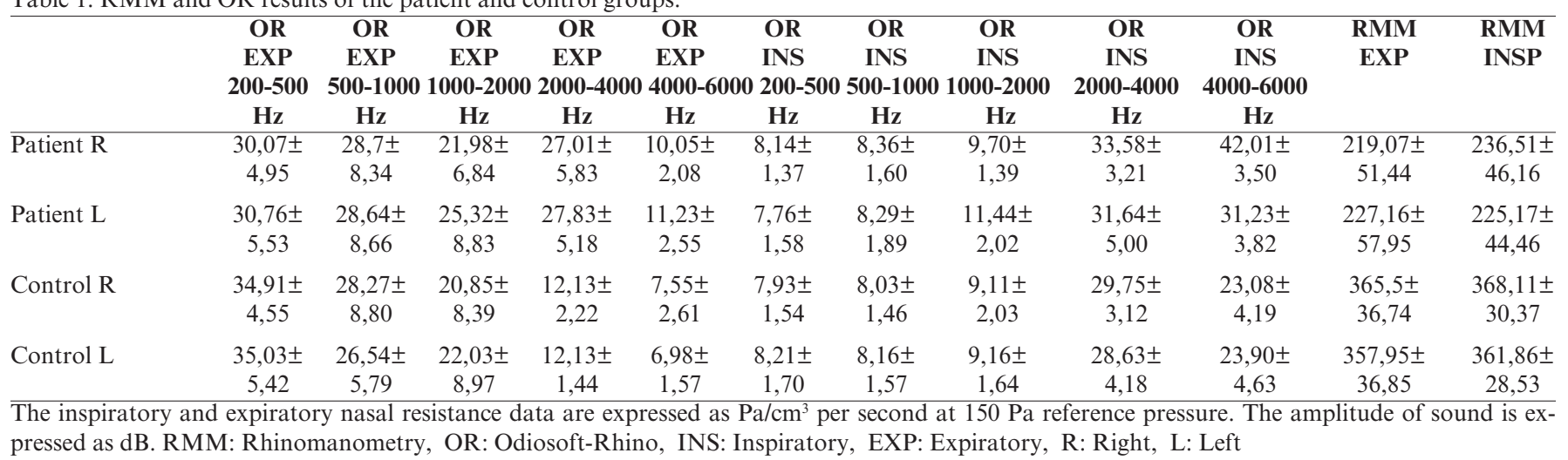


Table 2. The comparison of patient and control groups in terms of RMM and OR results.

\begin{tabular}{|c|c|c|c|c|c|c|c|c|c|c|c|c|c|}
\hline \multirow{4}{*}{$\begin{array}{l}\text { Patient } \\
\text { VS } \\
\text { Control }\end{array}$} & & OR EXP & OR EXP & OR EXP & OR EXP & OR EXP & OR & OR & OR & OR & OR & RMM & RMM \\
\hline & & 200 & $500-$ & $1000-$ & 2000 & $4000-$ & INS & INS & INS & INS & INS & EXP & INS \\
\hline & & $500 \mathrm{~Hz}$ & $1000 \mathrm{~Hz}$ & $2000 \mathrm{~Hz}$ & $4000 \mathrm{~Hz}$ & $6000 \mathrm{~Hz}$ & $200-$ & 500- & $1000-$ & $2000-$ & $4000-$ & & \\
\hline & & & & & & & $500 \mathrm{HZ}$ & $1000 \mathrm{HZ}$ & $2000 \mathrm{~Hz}$ & $4000 \mathrm{~Hz}$ & $6000 \mathrm{~Hz}$ & & \\
\hline \multirow[t]{2}{*}{$\overline{\mathrm{R}}$} & $\mathrm{t}$ & $-5,760$ & 0,283 & 0,844 & 19,513 & 6,038 & 0,827 & 1,236 & 1,904 & 6,844 & 27,639 & $-18,740$ & $-18,893$ \\
\hline & $\mathrm{p}$ & 0,000 & 0,778 & 0,400 & 0,000 & 0,000 & 0,410 & 0,219 & 0,060 & 0,000 & 0,000 & 0,000 & 0,000 \\
\hline \multirow[t]{2}{*}{$\mathrm{L}$} & $\mathrm{t}$ & $-4,412$ & 1,638 & 2,097 & 23,980 & 11,491 & $-1,548$ & 0,422 & 6,960 & 3,679 & 9,731 & $-15,449$ & $-20,987$ \\
\hline & $\mathrm{p}$ & 0,000 & 0,104 & 0,038 & 0,000 & 0,000 & 0,124 & 0,674 & 0,000 & 0,000 & 0,000 & 0,000 & 0,000 \\
\hline
\end{tabular}

RMM: Rhinomanometry, OR: Odiosoft-Rhino, INS: Inspiratory, EXP: Expiratory, R: Right, L: Left

\section{RESULTS}

OR and RMM were performed on 68 patients ( 32 female and 36 male; $47.0 \%$ vs $53.0 \%$, respectively) who had a nasal septal deviation and on a healthy control group of 61 subjects (28 females, 33 males; $45.9 \%$ vs $54.1 \%$, respectively). The age of the patients ranged between 18 and 54 (mean value $32.50 \pm 10.07$ ) and the control group's age ranged between 17 and 56 (mean of $34.36 \pm 11.18$ ). There was no significant difference between males and females ( $p>$ 0.05 ). All of the patients had a body mass index of 22 to 25. VAS of nasal obstruction was between 6 and 9 (mean value was $8.0 \pm 1.13$ ) for the deviation group and between 1 and $3(1.75 \pm 1.22)$ for the control group.

Endoscopic examinations of the patient and control groups were performed. The patients were classified as patients with septal deviation and healthy control group. Table 1 lists RMM and OR spectral analysis of expiratory and inspiratory sound intensity measurements of the patient and control group. When VAS of nasal obstruction for the deviation group was compared with the control group, a significant difference was found $(\mathrm{t}=30.14, \mathrm{p}<0.001)$.

Both RMM and OR were able to discriminate normal subjects from patients $(p<0.001)$. The nasal resistance values of RMM were significantly different between the patient and control groups on both inspiratory and expiratory examinations in both nasal cavities.

There were significant differences between patient and control

Table 3. Correlations between RMM, OR, Nasal endoscopy and VAS

\begin{tabular}{lccc} 
& & $\mathrm{r}$ & $\mathrm{p}$ \\
Left Endoscopic Findings & VAS & 0.693 & 0.000 \\
Right Endoscopic Findings & VAS & 0.597 & 0.000 \\
Left Inspiratory 2000-4000 Hz OR & VAS & 0.753 & 0.000 \\
Right Inspiratory 2000-4000 Hz OR & VAS & 0.617 & 0.000 \\
Left Expiratory 2000-4000 Hz OR & VAS & 0.642 & 0.000 \\
Right Expiratory 2000-4000 Hz OR & VAS & 0.715 & 0.000 \\
Left Inspiratory RMM & VAS & 0.562 & 0.000 \\
Right inspiratory RMM & VAS & 0.582 & 0.000 \\
Left Expiratory RMM & VAS & 0.515 & 0.000 \\
Right expiratory RMM & VAS & 0.563 & 0.000 \\
\hline RMM: Rhinomanometry & & &
\end{tabular}

RMM: Rhinomanometry

OR: Odiosoft-Rhino

VAS: Visual analogue scale of nasal obstruction groups in the OR at $200-500 \mathrm{~Hz}$ on expiration, at $1000-2000$ $\mathrm{Hz}, 2000-4000 \mathrm{~Hz}$ and $4000-6000 \mathrm{~Hz}$ on both the expiration and inspiration in the left side. There were also significant differences between patient and control groups in the OR at 200$500 \mathrm{~Hz}$ on expiration, at $2000-4000 \mathrm{~Hz}, 4000-6000 \mathrm{~Hz}$ on both the expiration and inspiration in the right side. Table 2 shows the comparison of RMM and OR results at each frequency interval of nasal expiratory and inspiratory sound amplitudes between patient and control groups. In the patient group, no significant difference among OR and RMM results were found according to the location of the nasal septal deviation. The correlations found are shown in Table 3.

\section{DISCUSSION}

The mechanics of nasal ventilation are based on the laws of fluid dynamics ${ }^{(5)}$. Measuring the transnasal pressure, nasal airflow volume and the ratio of $\Delta \mathrm{p} / \Delta \mathrm{V}$, and the nasal resistance with RMM gives values about how hard it is to breathe through the nose. Furthermore, nasal resistance is an internationally accepted index of nasal patency ${ }^{(6)}$. The thermoreceptors located in the vestibule ${ }^{(7)}$, the ratio between turbulent flow and laminar flow, velocity and loss of acceleration ${ }^{(8)}$ are thought to be factors in the reception of nasal patency.

Experiments found pure laminar flow with a very low velocity of about $20 \mathrm{~cm}^{3} / \mathrm{s}$. As flow velocity increases, turbulent flow increases while laminar flow decreases. When the flow reaches about $500 \mathrm{~cm}^{3} / \mathrm{s}$, purely turbulent flow is observed ${ }^{(9)}$. Actually, the predominant flow pattern is a regime of varied disturbances pattern termed transitional ${ }^{(10)}$. After the inspired air enters the nose, it converges as it passes the nasal valve area, which is the main resistive segment of the nasal cavity. An increase in the cross sectional area results in turbulence. Similarly, during expiration, when air from the nasopharynx reaches the choanal opening, an increase in the cross sectional area occurs again resulting in turbulence ${ }^{(11)}$. Turbulent airflow creates louder sound waves ${ }^{(12)}$ and such sound increases with the airflow cause a marked upward shift in the frequencies of the nasal spectra ${ }^{(13)}$.

OR is a newly developed method to evaluate nasal sounds. It was suggested that inspiratory nasal sound frequency and amplitude is a function of turbulence and airflow. Expiratory nasal sound OR intervals of 2000-4000 Hz and 4000-6000 Hz correlate with acoustic rhinometry findings and rhinomanometry results in healthy people ${ }^{(14)}$. The use of OR in patients with 


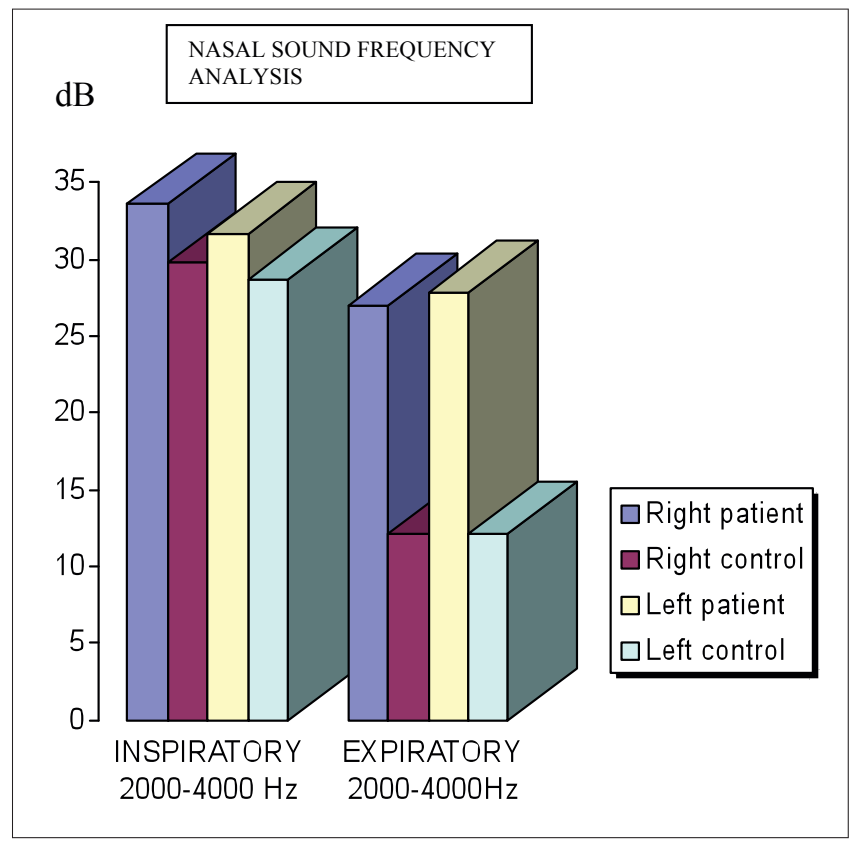

Figure 1. Mean expiratory and inspiratory nasal sound amplitude values at $2000-4000 \mathrm{~Hz}$ intervals which correlate with VAS are shown.

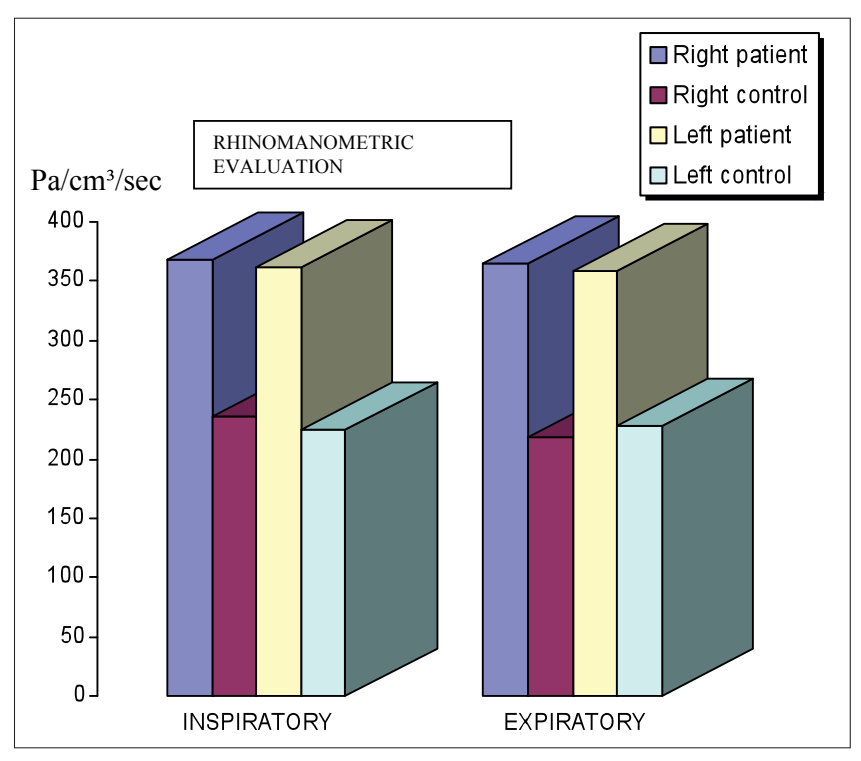

Figure 2. Mean nasal resistance which correlate with VAS are shown.

nasal septal deviation has been investigated by using acoustic rhinometry as a reference test showing a good correlation in expiratory $2000-4000 \mathrm{~Hz}$ and $4000-6000 \mathrm{~Hz}$ intervals (15). In these studies, OR was also shown to correlate with VAS ${ }^{(14,15)}$.

In this study, OR results were significantly different in patients with nasal septal deviation compared to the control group. Expiratory $2000-4000 \mathrm{~Hz}$ and $4000-6000 \mathrm{~Hz}$ signals were significantly different comparable to a previous study ${ }^{(15)}$. In contrast to other studies inspiratory $2000-4000 \mathrm{~Hz}$ and $4000-6000$ $\mathrm{Hz}$ signals and expiratory $500-1000 \mathrm{~Hz}$ range were also significantly different between both groups. It is very interesting that the inspiratory sound in the $2000-4000 \mathrm{~Hz}$ range showed a significant difference between patient and control groups.

The correlation between RMM and OR in patients with nasal septal deviation was investigated. Significant correlations between expiratory and inspiratory nasal sound at the interval of $2000-4000 \mathrm{~Hz}$ and VAS, between RMM at inspiration and expiration and VAS for both nasal cavities, between VAS and nasal endoscopic findings of both nasal cavities were found. No correlation between OR and RMM results could be found. However, since VAS is an indicator of sensation of nasal patency, OR can still be regarded as an indicator of nasal patency.

OR is a rapid, non-invasive test and requires little patient cooperation. It is very cheap and requires little performer education. The possibility to save the data digitally and to share over networks allows objective evaluation and screening of patients even when a specialist is not present in a distant area. RMM is a more complex test that lasts longer (a minimum of 5 minutes), requires more patient cooperation and is not widely available. The use of a nasal mask predisposes the nasal valve area to distortion.

Areas remaining to be investigated include pediatric nasal patency evaluation, measurement of outcome after rhinological operations, evaluation of patients with sinonasal polyposis, septal perforation and sleep problems.

\section{CONCLUSION}

OR may be an efficient method to evaluate nasal patency. It is more rapid and practical than RMM. It seems that sound intensity values in the $2000-4000 \mathrm{~Hz}$ frequency interval are more useful than other sound intensity intervals. However, showing a correlation between a test and a variable is not the same as proving that it is a useful diagnostic test. Further studies to determine the cut-off points for normal values, sensitivity and specificity should be planned and executed. Being noninvasive, it may be used as a clinical test during daily clinical practice. However, more studies with wide series are needed.

\section{ACKNOWLEDGMENTS}

We would like to thank Prof. Ahment Dirican, MD (Istanbul University, Cerrahpasa Medical School, Biostatistics Department, Istanbul, Turkey) for his invaluable scientific support and help.

\section{REFERENCES}

1. Pasterkamp H, Kraman SS, Wodicka GR. Respiratory sounds. Advances beyond the stethoscope. Am J Respir Crit Care Med. 1997; 156 (3 Pt 1): 974-987.

2. Rembold CM, Suratt PM. Children with obstructive sleep-disordered breathing generate high-frequency inspiratory sounds during sleep. Sleep 2004; 27: 1154-1161.

3. Polat H, Güler I. A simple computer-based measurement and analysis system of pulmonary auscultation sounds. J Med Syst. 2004; 28: 665-672.

4. Fiz JA, Jané R, Homs A, et al. Detection of wheezing during maximal forced exhalation in patients with obstructed airways. Chest 2002; 122: 186-191. 
5. Clement PA, Hirsch C. Rhinomanometry - a review. ORL J Otorhinolaryngol Relat Spec. 1984; 46: 173-191.

6. Clement PAR. Committee report on standardization of rhinomanometry. Rhinology 1984; 22: 151-155.

7. Tomkinson A, Eccles R. Comparison of the relative abilities of acoustic rhinometry, rhinomanometry, and the visual analogue scale in detecting change in the nasal cavity in a healthy adult population. Am J Rhinol. 1996; 10: 161-165.

8. Naito K, Kondo Y, Ohoka E, et al. New aerodynamic aspects of nasal patency. Rhinology 1995; 33: 26-29.

9. Mlynski G, Grützenmacher S, Plontke S, et al. Correlation of nasal morphology and respiratory function. Rhinology 2001; 39: 197-201.

10. Jaeger MJ, and Matthys $\mathrm{H}$. The pattern of flow in the upper human airways. Respir Physil. 1969; 6: 113-127.

11. Clement PA, Gordts F. Standardisation Committee on Objective Assessment of the Nasal Airway, IRS, and ERS. Consensus report on acoustic rhinometry and rhinomanometry. Rhinology 2005; 43: 169-179.

12. Moin P, Bewley T. Feedback control of turbulence. Appl Mech Rev. 1994; 47: 3-13.

13. Charbonneau G, Sudraud M, Soufflet G. Method for the evaluation of flow rate from pulmonary sounds. Bull Eur Physiopathol Respir. 1987; 23: 265-270.
14. Tahamiler R, Edizer DT, Canakcioglu S, et al. Odiosoft-Rhino versus rhinomanometry in healthy subjects. Acta Otolaryngol. 2007; 18: 1-5.

15. Tahamiler R, Canakcioglu S, Yilmaz S, et al. Expiratory nasal sound analysis as a new method for evaluation of nasal obstruction in patients with nasal septal deviation: comparison of expiratory nasal sounds from both deviated and normal nasal cavity. J Laryngol Otol. 2008; 122: 150-154

Yalçin Alimoglu

Cerrahpasa Burun Bogaz Hastaliklari Anabilim Dali

Cerrahpasa

Istanbul

Turkey

Tel: +90-533-642 1502

Fax: +90-212-586 1575

E-mail: alimoglu2001@gmail.com

\section{ADVANCES IN RHINOPLASTY}

one of the world's premier rhinoplasty courses held every two years

- attended by hundreds of surgeons from more than 40 countries

- world renowned faculty chosen for their expertise and experience in rhinoplasty

ample time for in-depth discussions

- dynamic and entertaining panels

- how I do it video sessions

- opportunities to present your challenging cases to the "panel of experts"

- hands-on cadaver dissection lab with entire faculty

sty

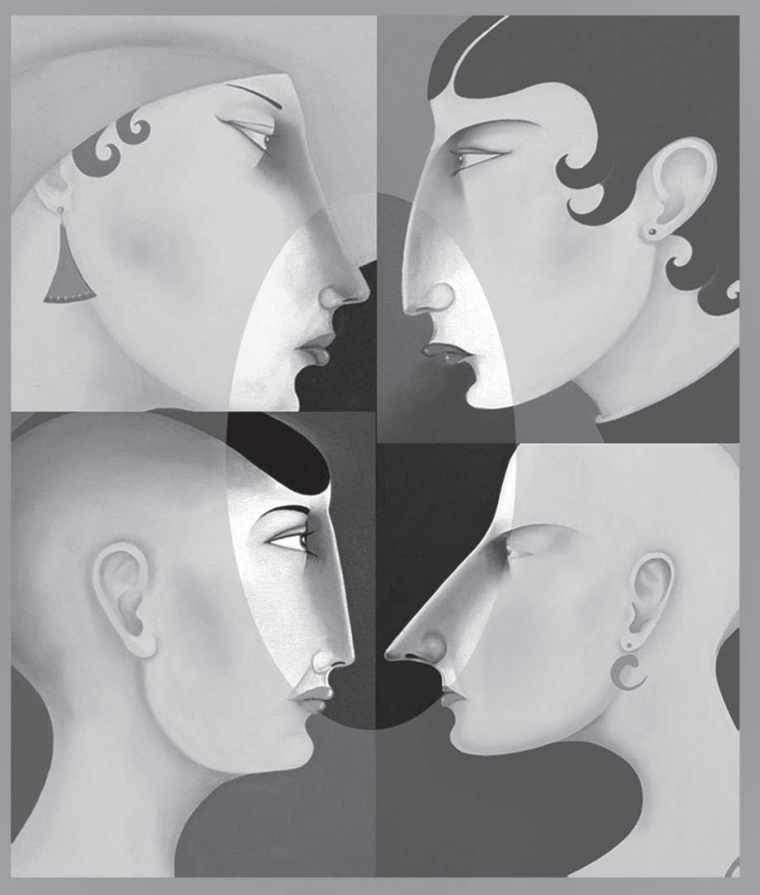

Sheraton Chicago Hotel and Towers
MAY 4-7, 2011 • CHICAGO, IL
Co-chairs: Stephen W. Perkins, MD Stephen S. Park, MD Minas Constantinides, MD

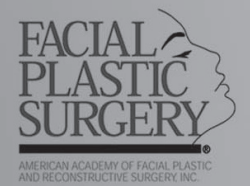

The Educational and Research Foundation for the American Academy of Facial Plastic and Reconstructive Surgery is accredited by the Accreditation Council for Continuing Medical Education (ACCME) to sponsor continuing medical education.

The full program is available on-line. www.aafprs.org info@aafprs.org 